1 Universidade de São Paulo (USP) - São Paulo (SP),

Brasil.

cpscesar@usp.br

2 Universidade Federal do Rio Grande do Sul (UFRGS)

- Porto Alegre (RS), Brasil.

\section{Uma avaliação dos efeitos do PMAQ-AB nas internações por condições sensíveis à Atenção Básica}

\author{
An evaluation of $P M A Q-A B$ effects on hospitalization for conditions \\ susceptible to Primary Care
}

César Soares ${ }^{\mathbf{1}}$ Marília Ramos²

DOI: $10.1590 / 0103-1104202012609$

RESUMO O objetivo deste artigo foi avaliar o efeito do Programa Nacional de Melhoria do Acesso e da Qualidade da Atenção Básica (PMAQ-AB) no acesso e na qualidade da Atenção Básica. As internações por condições sensíveis à Atenção Básica foram utilizadas como variável dependente e indicador indireto da qualidade e do acesso a esse nível de atenção. Utilizou-se o método quantitativo de estudo, tendo como unidade de análise todos os municípios brasileiros. O estudo se dividiu em duas fases: na primeira, foi realizada uma análise descritiva exploratória em série temporal sobre os municípios brasileiros, abrangendo o período de 2010 a 2014; e a segunda fase se caracterizou por avaliar o efeito do Programa, durante o mesmo período, no acesso e na qualidade da Atenção Básica, por meio da técnica estatística de regressão com dados de contagem. Os resultados, analisados por região, apontaram que o Programa está apresentando um efeito significativo na qualidade e no acesso da Atenção Básica, principalmente na região Nordeste. Este artigo surge como um importante informativo sobre as políticas de saúde no Brasil e, também, como um meio de instrumentalizar os gestores e os diversos atores envolvidos no desenvolvimento e discussão de um dos principais programas da Atenção Básica.

PALAVRAS-CHAVE Hospitalização. Avaliação em saúde. Atenção Primária à Saúde.

\begin{abstract}
The aim of this article was to evaluate the effect of the National Program for Access and Quality Improvement in Primary Care (PMAQ-AB) on access and quality of Primary Care. Hospitalization for conditions susceptible to Primary Care were used as a dependent variable and indirect indicator of quality and access to that level of care. The quantitative method of study was applied, adopting all Brazilian municipalities as unit of analysis. The study was divided into two phases. The first one performed an exploratory descriptive time series analysis on the Brazilian municipalities for the period from 2010 to 2014. The second phase was characterized for evaluating the effect of the Program, during the same period, on access and quality of Primary Care by means of the statistical regression technique with counting data. The results, analyzed by region, showed that the Program exerts a significant effect on the quality and access of Primary Care, especially in the Northeast region of Brazil. This article contains an important report on health policies in Brazil, and is a mean of instructing managers and the various actors involved in the development and discussion of one of the main programs in Primary Care.
\end{abstract}

KEYWORDS Hospitalization. Health evaluation. Primary Health Care. 


\section{Introdução}

O Ministério da Saúde (MS), procurando melhorar o nível de qualidade e acesso dos serviços de saúde oferecidos aos cidadãos pela Atenção Básica (AB), instituiu, no ano de 2011, o Programa Nacional de Melhoria do Acesso e da Qualidade da Atenção Básica (PMAQ$A B)$. Este é organizado em ciclos, estando, atualmente, no seu terceiro ciclo.

A área de abrangência do PMAQ, no seu primeiro ciclo, foi de 3.965 municípios, envolvendo um total de 17.203 equipes da AB. Entre elas, 17.165 equipes foram certificadas e habilitadas a receber os incentivos do programa. O investimento do Governo Federal foi de R\$ 70 milhões em 2011 e de R 700 milhões em 2012. Já no segundo ciclo, participaram do programa 30.522 equipes da $\mathrm{AB}, 19,7 \mathrm{mil}$ Equipes de Saúde Bucal (ESB), 1,8 mil Núcleos de Apoio às equipes de Saúde da Família (Nasf) e 857 Centros de Especialidades Odontológicas (CEO), entre 5.211 municípios. Foi investido $\mathrm{R} \$ 1,7$ bilhão em 2013, e mais de 2,5 bilhões em 20141. No primeiro ciclo, o MS estabeleceu um limite de equipes que poderiam participar do programa, fato inexistente no segundo ciclo, que incorporou, além disso, as ESB, os Nasf e os CEO' ${ }^{1}$. É importante destacar que esta pesquisa contemplou apenas os dois primeiros ciclos, que ocorreram, respectivamente, entre 2011 e 2012, e entre 2013 e 2014.

Para alcançar os seus objetivos, esse programa se organizou a partir de um conjunto de estratégias de qualificação, acompanhamento e avaliação do trabalho das equipes de saúde. Foram definidos, previamente, indicadores capazes de observar padrões de acesso e qualidade na $\mathrm{AB}$ que, posteriormente, serviram de parâmetro para a avaliação final. Estes se apresentam nas dimensões de infraestrutura, gestão para o desenvolvimento da $\mathrm{AB}$, valorização do trabalhador, acesso e qualidade da atenção à saúde e satisfação do usuário'.

A participação no PMAQ é voluntária, assim, o município, com as suas equipes da $\mathrm{AB}$, tem a autonomia para definir se irá aderir ou não ao programa; que tem um componente de incentivo à adesão, assegurando um repasse inicial de $20 \%$ do valor total previsto para os territórios que aderirem. A transferência restante dos recursos está vinculada ao desempenho de cada equipe, podendo atingir $100 \%$ do repasse ou a suspensão deste'.

O PMAQ, sendo um programa da $A B$, apresenta-se como um instrumento importante para esse nível de atenção, sobretudo, ao propor a melhora da qualidade e do acesso à saúde no Brasil. Isso porque o trabalho realizado na $A B$ é capaz de produzir efeitos positivos à saúde da população, como: diminuir as desigualdades sociais em saúde ao proporcionar um maior acesso aos serviços; aumentar a qualidade do atendimento enfocando as suas ações na prevenção e na promoção de saúde; tratar precocemente os problemas relativos à saúde; e reduzir os cuidados especializados. Consequentemente, esse nível de atenção está relacionado com a ocorrência de menores taxas de mortalidade por qualquer causa, além de um aumento na expectativa de vida dos indivíduos e da sua autopercepção acerca da própria saúde².

A AB, dentro de um contexto mais amplo de assistência, representa um nível de atenção cuja estratégia e recursos apontam para um maior e diversificado acesso aos serviços de saúde, visto que as equipes desse nível estão mais propensas a prestar um cuidado contínuo e integral. Ao apresentar um leque de atuação mais abrangente, caracterizando-se por apresentar ações que visam à promoção de saúde, à prevenção de agravos, ao tratamento e à reabilitação, contribui para a diminuição das taxas de hospitalização e utilização dos serviços de emergência, reduzindo os gastos gerais com os serviços de saúde ${ }^{2}$. Além disso, existem evidências robustas de que os sistemas de saúde com orientação para a $\mathrm{AB}$, comparados com outros que não dão ênfase a esse nível de atenção, apresentam resultados que apontam para uma diminuição mais acentuada da mortalidade e um acesso mais amplo aos serviços preventivos. Características 
importantes, pois apresentam a capacidade de contribuir para a diminuição das inequidades em saúde ${ }^{3}$.

Considerando que o PMAQ é uma das ações mais importantes, não só na esfera da $\mathrm{AB}$, mas em todo o Sistema Único de Saúde (SUS), sendo reconhecido pelo MS como um programa que apresenta estratégias que buscam a reestruturação dos sistemas municipais de saúde, este trabalho, partindo da hipótese de que esse programa está contribuindo para o aumento do acesso e da qualidade da $\mathrm{AB}$, avaliou o efeito que o PMAQ produziu nessas características.

Compreende-se 'acesso' nesta pesquisa como acessibilidade. Ou seja, a adequação dos profissionais e dos recursos materiais utilizados nos serviços básicos de saúde, com as necessidades de saúde dos pacientes ${ }^{4}$. Já o conceito de 'qualidade' aplicado à melhoria da $\mathrm{AB}$ é observado neste artigo como um avanço na eficácia do serviço. Isto é, a capacidade de este oferecer um cuidado apto a contribuir, da melhor forma possível, para a melhora na condição de saúde do indivíduo e da população ${ }^{5}$. Nesse sentido, considerando que os componentes principais do PMAQ apontam para um aperfeiçoamento dos serviços da $A B$ que abarca a definição de acesso e qualidade acima, esta pesquisa parte do pressuposto que, caso isso ocorra, o efeito se refletirá na saúde da população. As Internações por Condições Sensíveis à Atenção Primária (ICSAP), nesse contexto, surge como um indicador capaz de captar essa situação ${ }^{6}$.

A ICSAP foi proposta a partir de estudos que objetivavam criar um indicador relacionado com a atividade hospitalar que fosse capaz de captar de forma efetiva o funcionamento da $\mathrm{AB}$ à saúde. Este se refere a um conjunto de doenças que acometem os indivíduos; em que, caso a ação da AB seja efetiva, as internações por estas causas podem ser evitadas. Nesse sentindo, esse indicador surge como uma forma indireta de avaliar a qualidade do serviço e o acesso à $\mathrm{AB}$ do SUS6.

Com base na hipótese elencada acima, coloca-se que o resultado esperado por este trabalho se caracteriza pela ocorrência de um efeito significativo do PMAQ na diminuição das ICSAP, visto que altas taxas de ICSAP estão associadas a deficiências na cobertura dos serviços e/ou a baixa resolutividade da AB para determinados problemas de saúde ${ }^{6}$.

A relação entre esse indicador e a acessibilidade e qualidade da $\mathrm{AB}$ pode ser evidenciada a partir da observação da diminuição dessas internações conectadas a características específicas da AB, como: a amplitude da cobertura da Estratégia Saúde da Família; a existência de clínicas de saúde em áreas com escassez profissional; e a realização de consultas na $\mathrm{AB}$ de forma contínua e integral7 ${ }^{7}$. Ao possibilitar medir indiretamente esses fatores, a ICSAP representa um indicador importante para avaliar a acessibilidade e a qualidade da AB. Nota-se, no entanto, que ambas as características são tratadas em conjunto ao utilizar esse indicador, não sendo possível afirmar até que ponto o efeito observado nas ICSAP está relacionado com a acessibilidade ou com a qualidade da $\mathrm{AB}$, ou com ambas 6,8. Apesar dessa característica, a utilização desse indicador, além de pactuada entre o MS e os municípios brasileiros, objetivando monitorar a $\mathrm{AB}$, foi realizada em diversos estudos ${ }^{7-11}$.

Partindo do problema de pesquisa representado pelo seguinte questionamento: 'o programa denominado PMAQ está apresentando algum efeito no acesso e na qualidade da AB?', a proposta de avaliar esse programa visa instrumentalizar os gestores e os diversos atores envolvidos no desenvolvimento e discussão desse programa. Compreendendo que o PMAQ se apresenta em constante processo de aprimoramento, visto que ocorre por meio de ciclos, a avaliação deste se configura uma fonte importante de informação, que pode contribuir para possíveis reformulações e aperfeiçoamentos de ciclos posteriores. Por fim, esta pesquisa, apoiando-se no indicador ICSAP, visou contribuir para mais uma perspectiva avaliativa desse programa: o efeito dessa ação pública na saúde da população. 


\section{Metodologia}

O estudo, caracterizado por ter utilizado o método quantitativo, apresentou as ICSAP dos municípios brasileiros como um indicador indireto da qualidade e do acesso à $\mathrm{AB}$ de saúde. Este foi composto por duas fases, uma se distingue por ser descritiva exploratória em série temporal das ICSAP no Brasil e regiões, abrangendo o período de 2010 a 2014; sendo 2010 o ano base, isto é, período em que o PMAQ não tinha sido implementado. Os anos de 2011 e 2012, o primeiro ciclo do programa, e o período de 2013 a 2014, o segundo ciclo. Já a segunda fase distinguiu-se por ser um estudo ecológico, abarcando o mesmo período anterior, 2010 a 2014, com o intuito de observar o efeito do PMAQ nas ICSAP.

É importante destacar que a participação do município no PMAQ, no primeiro ciclo, não indicou, necessariamente, a inclusão de todas as equipes da $A B$ existentes nesse local, uma vez que o MS limitou o número de equipes que poderiam participar do programa nessa etapa. Assim, nem todas as equipes que desejavam aderir puderam fazê-lo; indicando, portanto, um possível viés positivo em relação a quais equipes participaram, já que existe a possibilidade de as 'melhores equipes' do município terem sido selecionadas de forma intencional. Além disso, não foi possível controlar esse efeito no modelo estatístico proposto nesta pesquisa, visto que a variável referente ao número de equipes que aderiram ao PMAQ no primeiro e segundo ciclo apresentou uma relação colinear com a variável explicativa principal; sendo, portanto, excluída das análises por exigências metodológicas, já que esta roubaria o efeito daquela variável.

A pesquisa se apoiou em dados secundários, expostos no quadro 1 abaixo, apresentando como unidade de análise os municípios brasileiros. No total, foram considerados 5.556 municípios, apesar de, em 2014, o total dessas entidades no Brasil ter sido igual a 5.570. Dezesseis municípios foram retirados da análise pelo seguinte motivo: foram reconhecidos novos municípios pela União em 2012, um dos períodos abordados na pesquisa. Com o intuito de facilitar as análises estatísticas, optou-se por desconsiderar esses novos municípios e excluir aqueles que existiam, mas sofreram alterações em seu território por causa da nova divisão de áreas.

No entanto, com exceção desses 16 municípios, $99,71 \%$ das entidades brasileiras foram consideradas na pesquisa. Dessa forma, foi considerado nas análises que o universo total de territórios foi abordado, não se caracterizando como uma amostra. Além disso, é importante acrescentar que as análises abarcaram a população brasileira, de 0-79 anos, de todos os 5.556 municípios, e examinaram os dados por país e região: ou seja, respectivamente, Brasil, Norte, Nordeste, Sudeste, Sul e CentroOeste. Essa faixa etária foi estabelecida uma vez que pesquisas que utilizaram a mesma variável dependente ${ }^{6,12}$ consideraram idades que variaram de 0-79 anos, já que, após essa idade, as internações não apresentaram correlações importantes com os procedimentos relacionados com a $\mathrm{AB}^{6}$. Para os modelos de análise, visando controlar os efeitos por faixas etárias, optou-se por estratificar as idades nos seguintes grandes grupos etários: 0-4, 5-19, 20-59 e 60-79 anos.

Para obter os subsídios necessários para a construção das variáveis, foram coletadas informações secundárias de sites que disponibilizam dados públicos. Estes foram expostos no quadro 1 abaixo. As informações relativas às ICSAP, variável dependente utilizada neste trabalho, foram obtidas por meio do site 'Datasus', em que aquelas estão disponíveis a partir da Autorização de Internação Hospitalar (AIH). Este é um documento utilizado para alimentar o Sistema de Informações Hospitalares do Sistema Único de Saúde (SIHSUS) e o responsável por liberar a internação de cada paciente. Existem dois tipos de AIH: a AIH-1, caracterizada por ser o tipo normal, sendo a sua emissão exclusiva dos gestores municipais ou estaduais do SUS; e a AIH-5, com caráter complementar, referente às internações de 
longa permanência, como as psiquiátricas ou doenças crônicas. No presente estudo, ante o caráter complexo que envolve a AIH-5, foi observada apenas a AIH-1 ${ }^{13}$.

Uma característica importante da AIH-1 no estudo sobre as ICSAP é que ela oferece a Classificação Internacional de Doenças 10 (CID-10), referente ao motivo pelo qual a pessoa foi internada. Assim, visto que a lista de ICSAP é composta por diagnósticos, esse dado se torna crucial. Esta é constituída por 120 categorias da CID-10 e 15 subcategorias que representam 20 tipos de diagnósticos que são agrupados de acordo com as possibilidades de intervenção e a magnitude dos agravos ${ }^{6}$.

Adicionalmente, é importante informar que o programa computacional utilizado para o processamento dos dados e realização das análises estatísticas foi o Stata versão 12.0.

O quadro 1 abaixo exibe as variáveis dependente e independentes, acompanhadas das suas respectivas descrições, das fontes de onde foram coletados os dados e do ano de referência.

Quadro 1. Descrição das variáveis utilizadas na pesquisa, Brasil, 2010 a 2014

\begin{tabular}{|c|c|c|c|}
\hline Indicador & Descrição do Indicador & Fonte dos Dados & Ano \\
\hline \multicolumn{4}{|l|}{ Variável Dependente } \\
\hline Número de ICSAP & $\begin{array}{l}\text { Número total de Internações por Condições Sensíveis à } \\
\text { Atenção Primária por faixa etária e sexo. }\end{array}$ & SIH-Datasus ${ }^{\mathbf{1 4}}$ & $\begin{array}{l}2010 a \\
2014\end{array}$ \\
\hline \multicolumn{4}{|c|}{ Variáveis Independentes } \\
\hline Adesão ao PMAQ & $\begin{array}{l}\text { Variável qualitativa dicotômica, em que o número } 1 \\
\text { indica que o município aderiu ao PMAQ e o número } 0 \\
\text { informa que não aderiu. }\end{array}$ & DAB-PMAQ ${ }^{15}$ & $\begin{array}{l}2010 a \\
2014\end{array}$ \\
\hline $\begin{array}{l}\text { Porcentagem de } \\
\text { ICSAP em indivídu- } \\
\text { os do sexo femini- } \\
\text { no/masculino }\end{array}$ & $\begin{array}{l}\text { Cálculo: número total de ICSAP de indivíduos do sexo } \\
\text { feminino/masculino por ano, área geográfica e idade, } \\
\text { dividido pelo número total de ICSAP por ano, área geo- } \\
\text { gráfica e idade e, por último, multiplicado por } 100 \text {. }\end{array}$ & SIH-Datasus ${ }^{16}$ & $\begin{array}{l}2010 a \\
2014\end{array}$ \\
\hline Cobertura da $A B$ & $\begin{array}{l}\text { Cobertura populacional estimada pelas equipes de } \\
\text { atenção básica. Cálculo: número de Equipes de Saúde da } \\
\text { Família (ESF) mais o número de ESF equivalente, multi- } \\
\text { plicado por } 3.000 \text {, dividido pela população do mesmo } \\
\text { local e período, multiplicado por } 100 \text {. }\end{array}$ & Pactuaç̃̃es-DataSUS16 & $\begin{array}{l}2010 a \\
2014\end{array}$ \\
\hline $\begin{array}{l}\text { Número de leitos } \\
\text { por habitante }\end{array}$ & $\begin{array}{l}\text { Cálculo: número total de leitos disponíveis nos municí- } \\
\text { pios, dividido pela população total local. }\end{array}$ & $\begin{array}{l}\text { CNES-Datasus } \mathbf{1 7} \\
\text { Instituto Brasileiro de } \\
\text { Geografia e Estatística } \\
\text { (IBGE) }\end{array}$ & $\begin{array}{l}2010 a \\
2014\end{array}$ \\
\hline $\begin{array}{l}\text { Cobertura dos } \\
\text { planos de saúde }\end{array}$ & $\begin{array}{l}\text { Cálculo: número total de beneficiários de planos priva- } \\
\text { dos de saúde por município, ano e idade, dividido pela } \\
\text { população do mesmo local, período e idade, multiplicado } \\
\text { por } 100 \text {. }\end{array}$ & $\begin{array}{l}\text { Agência Nacional de } \\
\text { Saúde Suplementar } \\
\text { (ANS) } \mathbf{1 9}^{\mathbf{9}} \text { IBGE18 }\end{array}$ & $\begin{array}{l}2010 a \\
2014\end{array}$ \\
\hline Coeficiente de Gini & $\begin{array}{l}\text { Medida de desigualdade que oscila entre } 0 \text { e } 1 \text {, em que } 0 \\
\text { corresponde à completa igualdade em relação ao rendi- } \\
\text { mento e } 1 \text { corresponde à completa desigualdade. }\end{array}$ & PNUD20 & 2010 \\
\hline $\begin{array}{l}\text { População dos } \\
\text { municípios de 0-79 } \\
\text { anos }\end{array}$ & Número total de indivíduos por faixa etária e sexo. & IBGE18 & $\begin{array}{l}2010 a \\
2014\end{array}$ \\
\hline $\begin{array}{l}\text { Número de médi- } \\
\text { cos do programa } \\
\text { Mais Médicos por } \\
\text { mil habitantes }\end{array}$ & $\begin{array}{l}\text { Cálculo: total de médicos em atividade do programa } \\
\text { Mais Médicos, dividido pela população total do local, } \\
\text { multiplicado por } 1.000 \text {. }\end{array}$ & Região-Redes $\mathbf{2 1}$ & 2014 \\
\hline
\end{tabular}

Fonte: Elaboração própria. 
É importante pontuar que a variável independente 'adesão do município ao PMAQ' configura-se a mais importante, posto que será a partir dela que o efeito do programa será analisado. As variáveis 'porcentagem de ICSAP em indivíduos do sexo masculino/feminino’ foram incluídas nos modelos com o intuito de controlar os efeitos que ambas as questões podem apresentar nesse tipo de internação, como destaca o estudo que aprovou a lista das ICSAP no Brasil ${ }^{6}$. Essa informação foi utilizada separadamente para diferentes grupos etários.

As variáveis relativas aos serviços e estabelecimentos de saúde - cobertura da $A B$, número de leitos por habitante e cobertura dos planos de saúde - e às questões socioeconômicas - coeficiente de Gini e a população de 0-79 anos dos municípios - foram consideradas, visto que os estudos utilizando as ICSAP ressaltam a importância em observar esses dados ao analisar as internações ${ }^{6,12}$. Em função da disponibilidade de dados, destaca-se como uma limitação da análise a utilização do valor do coeficiente de Gini de 2010 para todos os anos analisados, exigindo cautela ao examinar os seus resultados.

Por último, o número de médicos, do programa Mais Médicos, por mil habitantes foi considerado por ser uma variável de confusão. Isso porque caracteriza-se como uma política pública que foi implementada em 2013 e que poderia influenciar a diminuição das ICSAP ao alocar médicos em regiões onde há escassez ou ausência desses profissionais na $A^{22}$.

É importante destacar a possível limitação na coleta de informações pelos órgãos responsáveis, indicados no quadro 1 , acerca dos municípios, exigindo uma análise cautelosa dos resultados conforme exposto nas seções seguintes deste artigo.

\section{Métodos de análise dos dados}

Para a análise de dados, foi ajustado um modelo de regressão multivariada para painel de dados, considerando os anos de
2010 a 2014, com resposta binomial negativa e modelos de efeitos fixos, controlado pela população de cada município por meio do comando offset. A regressão binomial negativa é indicada para analisar dados numéricos resultantes de contagem em situações em que há sobredispersão (over - dispersion) dos dados, isto é, quando os dados apresentam valores de variância superiores à média ${ }^{23}$, como é caso das ICSAP aqui analisadas. Além disso, essa técnica foi adotada por ser amplamente utilizada nos estudos que empregam as ICSAP como variável dependente ${ }^{\mathbf{1 2}}$.

O banco de dados em painel utilizado na análise se apresenta de forma balanceada, visto que há informações de todos os municípios sobre a variável dependente e independente principal durante os cinco anos. O modelo de efeitos fixos foi definido após a aplicação do teste de Hausman. Por fim, a associação entre a taxa de ICSAP e as covariáveis do estudo foi estimada pelas taxas de incidência das ICSAP, acompanhada de seus respectivos intervalos de confiança a $95 \%$.

\section{Resultados}

\section{Descrição das ICSAP}

Os gráficos apresentados, tanto em nível nacional como regional, foram construídos com o objetivo de comparar as ICSAP entre os municípios que aderiram e não aderiram ao PMAQ.

O gráfico 1 expõe como as taxas de ICSAP por dez mil habitantes diminuíram em ambos os grupos no decorrer dos anos. Contudo, no grupo que não aderiu ao PMAQ, foi possível observar que, na mudança de ciclo do programa, de 2012 para 2013, este apresentou um aumento nas internações. 
Gráfico 1. Taxas de ICSAP por 10 mil habitantes/ano, dos municípios que aderiram e não aderiram ao PMAQ, Brasil, 2010 a 2014

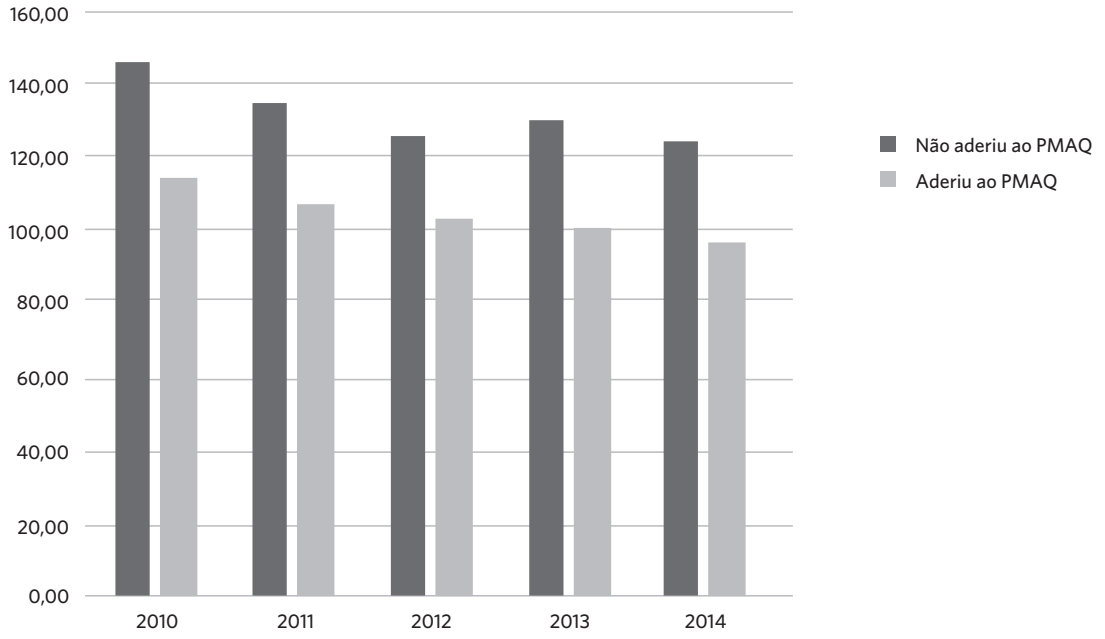

Fonte: SIH-Datasus ${ }^{\mathbf{1 4}}$, IBGE18 e Departamento de Atenção Básica (DAB)-PMAQ15.

Da mesma forma, abaixo foi exposto um gráfico similar sobre as ICSAP em nível regional.

Gráfico 2. Taxas de ICSAP por 10 mil habitantes/ano, dos municípios que aderiram e não aderiram ao $P M A Q$, regiões brasileiras, 2010 a 2014 250

não aderiu ao PMAQ

- Aderiu ao PMAQ

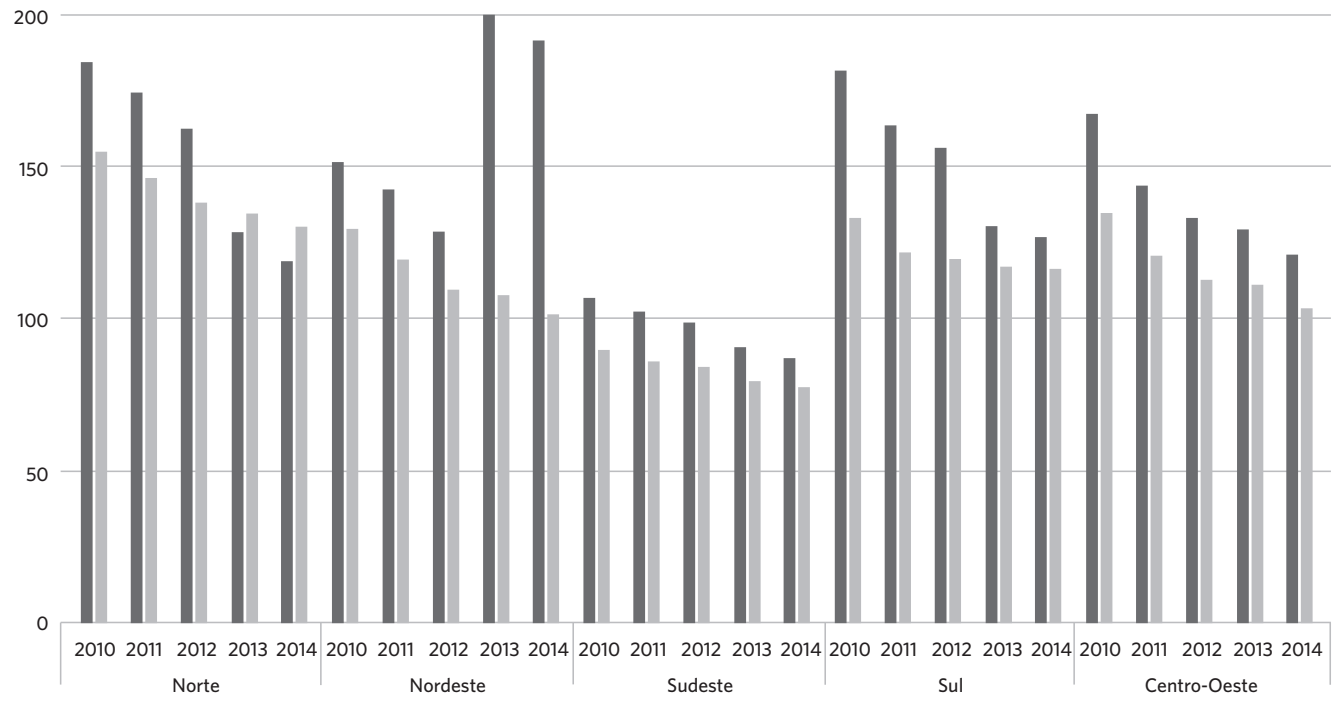

Fonte: SIH-Datasus ${ }^{\mathbf{1 4}}$ IBGE18 e Departamento de Atenção Básica (DAB)-PMAQ15. 
A partir do gráfico 2, foi possível notar que, em todos os anos, de 2010 a 2014, todas as regiões apresentaram taxas totais mais altas no grupo que não aderiu ao PMAQ. Com exceção da região Norte, em que, durante o segundo ciclo, 2013 e 2014, o grupo que aderiu ao programa apresentou uma taxa mais elevada do que o grupo que não aderiu. Além disso, o Nordeste, em um movimento contrário ao das outras regiões do País, apresentou um aumento abrupto nas ICSAP, a partir do segundo ciclo, nos municípios que não aderiram ao programa. Tal comportamento pode estar relacionado com o PMAQ, como foi avaliado na seção seguinte.

\section{O efeito do PMAQ nas ICSAP}

Na tabela 1 abaixo, na coluna referente ao Brasil, a taxa de incidência (IRR) do PMAQ indicou que os municípios que aderiram ao programa apresentaram, no decorrer de 2010 a 2014, 9\% a menos de ICSAP em relação àqueles que não aderiram, mantendo as outras variáveis constantes. Já em relação às variáveis 'cobertura dos planos de saúde' e 'médicos alocados pelo programa Mais Médicos', foi possível dizer que a primeira apresentou $1 \%$ de diminuição das ICSAP para cada unidade a mais da cobertura dos planos de saúde; já a segunda exibiu 35\% de diminuição das mesmas internações para cada unidade a mais de médicos do programa Mais Médicos por mil habitantes.
Em um sentido oposto, a variável 'número de leitos por habitante' apontou para um aumento da taxa de incidência no valor de $23 \%$ das ICSAP para cada unidade a mais de leito, mantendo as outras variáveis constantes. Já as variáveis relativas ao sexo/idade, assim como à cobertura da $\mathrm{AB}$, não apontaram resultados significantes. Por último, o índice de Gini: este apresentou um comportamento peculiar, visto que o seu valor, para todas as regiões, foi próximo de zero, indicando que esse indicador possivelmente não oscilou no decorrer do tempo. A causa de tal fato pode estar relacionada com a utilização do valor de 2010 do Gini em todos os anos, não apresentando, portanto, variação dos seus valores. Sendo assim, essa questão pode ter gerado algum viés, comprometendo a interpretação.

A coluna referente à região Norte apontou para o mesmo cenário do Brasil, mas com valores distintos: os municípios que aderiram ao PMAQ apresentaram 6\% menos ICSAP em relação àqueles que não aderiram; um aumento da taxa de incidência no valor de $8 \%$ das ICSAP para cada unidade a mais de leitos, mantendo as outras variáveis constantes; $2 \%$ de diminuição das ICSAP para cada unidade a mais na cobertura dos planos de saúde; e $45 \%$ de diminuição das mesmas internações para cada unidade a mais de médicos do programa Mais Médicos por mil habitantes.

Tabela 1. Resultados da regressão com dados de contagem estimados por meio das taxas de incidência das ICSAP e a sua associação com as covariáveis do estudo, Brasil e regiões brasileiras, 2010 a 2014

\begin{tabular}{lrrrrrr}
\hline Variáveis & \multicolumn{2}{c}{ Brasil } & \multicolumn{2}{c}{ Norte } & \multicolumn{2}{c}{ Nordeste } \\
\cline { 2 - 7 } & IRR & $\begin{array}{r}\text { Intervalo de } \\
\text { Confiança (95\%) }\end{array}$ & IRR & $\begin{array}{r}\text { Intervalo de } \\
\text { Confiança (95\%) }\end{array}$ & IRR & $\begin{array}{r}\text { Intervalo de } \\
\text { Confiança (95\%) }\end{array}$ \\
\hline PMAQ & 0,91 & $0,90-0,91$ & 0,94 & $0,91-0,96$ & 0,86 & $0,85-0,88$ \\
Internação 0-4 anos Feminino & 1,00 & $1,00-1,00$ & 1,00 & $1,00-1,01$ & 1,00 & $1,00-1,00$ \\
Internação 0-4 anos Masculino & 1,00 & $1,00-1,00$ & 1,01 & $1,00-1,01$ & 1,00 & $1,00-1,00$ \\
Internação 5-19 anos Feminino & 1,00 & $1,00-1,00$ & 1,00 & $1,00-1,00$ & 1,00 & $1,00-1,00$ \\
Internação 5-19 anos Masculino & 1,00 & $1,00-1,00$ & 1,00 & $1,00-1,01$ & 1,00 & $1,00-1,00$ \\
Internação 20-59 anos Feminino & 1,01 & $1,00-1,01$ & 1,01 & $1,01-1,02$ & 1,00 & $1,00-1,01$ \\
Internação 20-59 anos Masculino & 1,01 & $1,00-1,01$ & 1,01 & $1,01-1,02$ & 1,00 & $1,00-1,01$ \\
\hline
\end{tabular}


Tabela 1. (cont.)

\begin{tabular}{|c|c|c|c|c|c|c|}
\hline \multirow[t]{3}{*}{ Variáveis } & \multicolumn{2}{|r|}{ Brasil } & \multicolumn{2}{|r|}{ Norte } & \multicolumn{2}{|r|}{ Nordeste } \\
\hline & IRR & Intervalo de & IRR & Intervalo de & IRR & Intervalo de \\
\hline & \multicolumn{2}{|r|}{ Confiança $(95 \%)$} & \multicolumn{2}{|r|}{ Confiança $(95 \%)$} & \multicolumn{2}{|r|}{ Confiança (95\%) } \\
\hline Internação 60-79 anos Feminino & 1,01 & $1,00-1,01$ & 1,00 & $1,00-1,01$ & 1,00 & $1,00-1,01$ \\
\hline Internação 60-79 anos Masculino & 1,00 & $1,00-1,01$ & 1,00 & $1,00-1,00$ & 1,00 & $1,00-1,00$ \\
\hline Cobertura da $A B$ & 1,00 & $1,00-1,00$ & 1,00 & $1,00-1,00$ & 1,00 & $1,00-1,00$ \\
\hline Número de leitos por habitante & 1,23 & $1,16-1,30$ & 1,08 & $0,74-1,58$ & 0,95 & $0,83-1,08$ \\
\hline Cobertura dos planos de saúde & 0,99 & $0,99-0,99$ & 0,98 & $0,97-0,99$ & 0,99 & $0,99-1,00$ \\
\hline Gini & 0,00 & $0,00-0,00$ & 0,00 & $0,00-0,01$ & 0,00 & $0,00-0,01$ \\
\hline $\begin{array}{l}\text { Médicos do Mais Médicos por } \\
\text { mil habitantes }\end{array}$ & 0,65 & $0,61-0,69$ & 0,55 & $0,45-0,68$ & 0,64 & $0,57-0,71$ \\
\hline Constante & 0,01 & $0,01-0,02$ & 0,00 & $0,00-0,00$ & 0,00 & $0,00-0,01$ \\
\hline
\end{tabular}

Fonte: SIH-Datasus 14, IBGE18, DAB-PMAQ15, Pactuações-Datasus ${ }^{16}$, Cadastro Nacional de Estabelecimentos de Saúde (CNES)-Datasus ${ }^{17}$ ANS19, PNUD20 e Região-Redes 21

Taxa de incidência (IRR).

Na coluna referente ao Nordeste, os municípios que aderiram ao PMAQ apresentaram $14 \%$ menos ICSAP do que aqueles que não aderiram. Nesse caso, ocorreu uma diminuição na taxa de incidência no valor de $5 \%$ das ICSAP para cada unidade a mais de leito. A variável 'cobertura dos planos de saúde' apresentou 1\% de diminuição das ICSAP para cada unidade a mais nessa cobertura, e a variável referente ao programa Mais Médicos apontou 36\% de diminuição das ICSAP para cada unidade a mais de médicos do programa por mil habitantes. Já as outras variáveis relacionadas com a cobertura da $\mathrm{AB}$, idade/sexo e Gini seguiram o mesmo padrão dos modelos anteriores, sendo a explicação anterior válida, também, nesse caso.

A coluna relativa ao Sudeste, na tabela 2, exibiu o mesmo cenário observado no Brasil: os municípios que aderiram ao PMAQ apresentaram $5 \%$ menos ICSAP em relação àqueles que não aderiram; ocorreu um aumento na taxa de incidência no valor de $29 \%$ das ICSAP para cada unidade a mais de leitos, mantendo as outras vaiáveis constantes; $3 \%$ de diminuição das ICSAP para cada unidade a mais na cobertura dos planos de saúde; e $22 \%$ de diminuição das internações para cada unidade a mais de médicos do programa Mais Médicos por mil habitantes.

Tabela 2. Resultados da regressão com dados de contagem estimados por meio das taxas de incidência das ICSAP e a sua associação com as covariáveis do estudo, regiões brasileiras, 2010 a 2014

\begin{tabular}{lrrrrrr}
\hline Variáveis & \multicolumn{2}{c}{ Sudeste } & \multicolumn{2}{c}{ Sul } & \multicolumn{2}{c}{ Centro-Oeste } \\
\cline { 2 - 7 } & IRR & $\begin{array}{r}\text { Intervalo de } \\
\text { Confiança (95\%) }\end{array}$ & IRR & $\begin{array}{r}\text { Intervalo de } \\
\text { Confiança (95\%) }\end{array}$ & IRR & $\begin{array}{r}\text { Intervalo de } \\
\text { Confiança (95\%) }\end{array}$ \\
\hline PMAQ & 0,95 & $0,94-0,96$ & 0,92 & $0,91-0,93$ & 0,89 & $0,87-0,92$ \\
Internação 0-4 anos Feminino & 1,00 & $1,00-1,00$ & 1,00 & $1,00-1,00$ & 1,00 & $1,00-1,00$ \\
Internação 0-4 anos Masculino & 1,00 & $1,00-1,00$ & 1,00 & $1,00-1,00$ & 1,00 & $1,00-1,00$ \\
Internação 5-19 anos Feminino & 1,00 & $1,00-1,00$ & 1,00 & $1,00-1,00$ & 1,00 & $1,00-1,00$ \\
Internação 5-19 anos Masculino & 1,00 & $1,00-1,00$ & 1,00 & $1,00-1,00$ & 1,00 & $1,00-1,00$ \\
Internação 20-59 anos Feminino & 1,01 & $1,00-1,01$ & 1,00 & $1,00-1,01$ & 1,01 & $1,00-1,01$ \\
Internação 20-59 anos Masculino & 1,01 & $1,00-1,01$ & 1,00 & $1,00-1,01$ & 1,00 & $1,00-1,01$ \\
\hline
\end{tabular}


Tabela 2. (cont.)

\begin{tabular}{lrrrrrr}
\hline Variáveis & \multicolumn{2}{c}{ Sudeste } & \multicolumn{2}{c}{ Sul } & \multicolumn{2}{c}{ Centro-Oeste } \\
\cline { 2 - 7 } & IRR & $\begin{array}{r}\text { Intervalo de } \\
\text { Confiança (95\%) }\end{array}$ & IRR & $\begin{array}{r}\text { Intervalo de } \\
\text { Confiança (95\%) }\end{array}$ & $\begin{array}{r}\text { IRR } \\
\text { Intervalo de } \\
\text { Confiança (95\%) }\end{array}$ \\
\hline Internação 60-79 anos Feminino & 1,00 & $1,00-1,01$ & 1,00 & $1,00-1,01$ & 1,01 & $1,00-1,01$ \\
Internação 60-79 anos Masculino & 1,00 & $1,00-1,01$ & 1,00 & $1,00-1,01$ & 1,00 & $1,00-1,01$ \\
Cobertura da AB & 1,00 & $1,00-1,00$ & 1,00 & $1,00-1,00$ & 1,00 & $1,00-1,00$ \\
Número de leitos por habitante & 1,29 & $1,19-1,40$ & 1,11 & $1,00-1,24$ & 1,48 & $1,16-1,89$ \\
Cobertura dos planos de saúde & 0,97 & $0,97-0,97$ & 0,98 & $0,98-0,98$ & 0,99 & $0,98-0,99$ \\
Gini & 0,00 & $0,00-0,00$ & 0,01 & $0,00-0,01$ & 0,04 & $0,01-0,12$ \\
Médicos do Mais Médicos por & 0,78 & $0,69-0,89$ & 0,71 & $0,63-0,79$ & 0,78 & $0,59-1,04$ \\
mil habitantes & & $0,00-0,03$ & 0,01 & $0,00-0,06$ & 0,00 & $0,00-0,00$ \\
Constante & 0,01 & 0,000 & & & & \\
\hline
\end{tabular}

Fonte: SIH-Datasus 14, IBGE18, DAB-PMAQ15, Pactuaç̃̃es-Datasus ${ }^{16}$, Cadastro Nacional de Estabelecimentos de Saúde (CNES)-Datasus 17, ANS19, PNUD20 e Região-Redes 21

Taxa de incidência (IRR).

Por último, as regiões Sul e Centro-Oeste: na primeira, respectivamente, a presença do PMAQ nos municípios indicou $8 \%$ menos ICSAP em relação àqueles lugares nos quais o programa não existia; ocorreu um aumento na taxa de incidência no valor de $11 \%$ das ICSAP para cada unidade a mais de leitos; foi exibida uma diminuição de $2 \%$ das ICSAP para cada unidade a mais da cobertura dos planos de saúde; e uma diminuição de $29 \%$ das internações para cada unidade a mais de médicos do programa Mais Médicos por mil habitantes.

Já a região Centro-Oeste apresentou uma diminuição de $11 \%$ das ICSAP nos municípios que aderiram ao PMAQ em relação àqueles que não aderiram; um aumento da taxa de incidência no valor de $48 \%$ das ICSAP para cada unidade a mais de leito por habitante; $1 \%$ de diminuição das ICSAP para cada unidade a mais da cobertura dos planos de saúde; e $22 \%$ de diminuição das internações para cada unidade a mais de médicos, do programa Mais Médicos, por mil habitantes.

\section{Discussão}

Foi possível notar que o PMAQ exibiu um efeito significativo na redução das ICSAP em todas as regiões e, também, no Brasil. Além disso, a região Nordeste parece ser aquela que mais se beneficiou com esse programa. Diante dessa situação, é natural o questionamento sobre os fatores que contribuíram para que o Nordeste se destacasse em relação às outras regiões.

Uma estratégia para tentar responder a essa pergunta é considerar as peculiaridades inerentes ao Brasil, isto é, avaliar os resultados ponderando que o País apresenta uma variedade de características histórico-estruturais que afetam diretamente a configuração do SUS. Assim, foram analisadas as pesquisas que utilizaram dados coletados pelo PMAQ com o objetivo de examinar com mais detalhes as particularidades daqueles municípios que aderiram ao programa.

Considerando esse aspecto, um estudo ${ }^{24}$ apresentou como objetivo analisar a estrutura física das Unidades Básicas de Saúde (UBS) com base no conjunto de ambientes recomendado pelo Manual de Estrutura Física das UBS do MS, considerando os dados coletados dos municípios que aderiram ao primeiro e ao segundo ciclo do PMAQ. Os resultados apontam para os seguintes aspectos: considerando os ambientes recomendados no Manual, o consultório odontológico, a sala de curativos e procedimentos, a sala de vacina, a sala de nebulização e os 
sanitários aumentaram em todas as regiões, indicando uma melhora que abrangeu todo o País; sendo um dos fatores que podem ter contribuído para a diminuição nacional das ICSAP entre o período de 2010 e 2014.

No entanto, $\mathrm{o} \operatorname{artigo}^{\mathbf{2 4}}$ aponta que a região Nordeste foi o local onde as salas de vacina, as salas de curativos e procedimentos e as salas de reunião e atividades educativas apresentaram os maiores aumentos. Observou-se, também, nesta última região e no Norte, que as UBS com um a três e quatro a seis ambientes tiveram a sua maior redução entre os dois ciclos do PMAQ, em comparação com as outras regiões. Ademais, complementarmente, que as unidades com sete a nove ambientes aumentaram em maior grau, também, nessas duas regiões.

Nesse contexto, um estudo ${ }^{25}$ apontou que a sala de reunião possibilita um espaço importante nas UBS, voltado para a realização de atividades que transpassam os atendimentos clínicos, contribuindo para o aperfeiçoamento de ações programáticas e gestão do serviço. Indo no mesmo sentido, outro artigo ${ }^{26}$ destaca que a ausência desse espaço pode obstaculizar a ocorrência de atividades coletivas com a comunidade e a existência de planejamento e avaliação das ações dos profissionais das equipes de saúde. Nesse sentido, a substituição gradativa das UBS com menos capacidade por espaços mais adequados pode indicar uma melhora da atenção à população.

É importante pontuar que o Sul se destacou por ser o território onde essas características se apresentaram em maior quantidade durante os anos do estudo, contudo, o aumento desses subsídios de um ciclo do PMAQ para o outro ocorreu de forma mais intensa apenas no Nordeste ${ }^{24}$. De forma mais clara, o aumento aqui não indica que a região apresentou uma quantidade mais adequada dos fatores descritos, mas a exibição de uma oscilação positiva no período de 2011 a 2014.

Similarmente, outro estudo ${ }^{27}$, a partir da construção de uma tipologia da estrutura das UBS utilizando os dados do primeiro ciclo do PMAQ, considerando dimensões relacionadas com os tipos de equipe, elenco de profissionais, turnos de funcionamento, serviços disponíveis e instalações e insumos, apontou o seguinte cenário: as piores estruturas se encontravam, proporcionalmente, no Norte, e em valores absolutos, nas regiões Norte e Nordeste. Já as melhores localizavam-se em maior número nas regiões Sul e Sudeste. Considerando o destaque da região Nordeste nesta pesquisa, e que os dados observados neste estudo ${ }^{\mathbf{2 7}}$ se referem ao primeiro ciclo do PMAQ, é possível supor que esse programa contribuiu para a melhora dessa estrutura de um ciclo para o outro.

Em consonância com esses estudos, uma pesquisa ${ }^{28}$ buscou observar a influência da estrutura das UBS e do processo de trabalho das equipes de saúde nas ICSAP. Os dados foram retirados das avaliações realizadas no primeiro ciclo do PMAQ. Os resultados apontaram que uma maior cobertura dos programas de distribuição de renda, um maior percentual de UBS funcionando em horário mínimo e a maior disponibilidade de vacinas estão significativamente relacionadas com a diminuição das internações nos municípios. É importante ressaltar aqui a disponibilidade de vacinas como um fator que está relacionado com a diminuição das ICSAP, visto que esse foi justamente um dos componentes que se destacaram no Nordeste ao comparar com as outras regiões.

Em suma, essas distintas melhoras, com destaque na região Nordeste, parecem ter contribuído para a proeminência desse local em relação ao efeito do PMAQ nas ICSAP. Contudo, é importante pontuar que, apesar desse aumento e dos efeitos observados, o artigo $^{24}$ coloca que as UBS ainda não apresentam um padrão ideal de funcionamento como recomendado pelo MS.

Em confluência com o destaque da região Nordeste em determinadas características, outro artigo ${ }^{29}$ parece corroborar essa situação. O objetivo deste foi descrever as condições de produção e registro das informações geradas nas UBS mediante indicadores obtidos por meio dos dados do primeiro ciclo do PMAQ. Estes se referiram às condições de infraestrutura e 
informática, à educação permanente e ao processo de trabalho. Assim, ao analisar os dados das grandes regiões, delimitadas pelo artigo como Norte e Nordeste, Sul e Sudeste e CentroOeste, foi possível observar que, em comparação com as outras, a primeira, respectivamente, apresentou um valor superior no indicador relacionado com o processo de trabalho, em específico, o monitoramento e a análise dos indicadores e informações de saúde.

Tal fato sugere uma preocupação das equipes da $\mathrm{AB}$ com o resultado das suas ações na saúde da população, possibilitando um posicionamento crítico diante da atuação profissional e um aperfeiçoamento constante dos atos realizados. Esses dados, que são observados por meio do Sistema de Informação da Atenção Básica (Siab), permitem a observância, inclusive, de informações relacionadas com as ICSAP. Sendo assim, uma maior vigilância e consideração desses dados poderia influenciar as equipes dessa região a aperfeiçoarem a atenção e, consequentemente, diminuírem as ICSAP de forma mais expressiva.

Dessa forma, apesar de o artigo ${ }^{29}$ apresentar o resultado referente à região Nordeste em conjunto com a Norte, ao considerar os outros dados expostos acima, o Nordeste surge como o local onde o PMAQ parece ter proporcionado um maior acesso e melhor qualidade de serviço para a população.

Nesse contexto, é importante ressaltar, também, os efeitos observados das outras variáveis independentes nas ICSAP. Uma delas foi a cobertura dos planos de saúde. Essa variável apontou que os territórios que apresentaram mais internações foram aqueles em que esse fator esteve menos presente. Esses dados vão ao encontro dos outros encontrados na literatura sobre às ICSAP'2. A cobertura do plano de saúde no Brasil representa um importante indicador de acesso ${ }^{30}$, evidenciando a possível razão de ela estar negativamente relacionada com as ICSAP nos resultados dos modelos. Ou seja, quanto maior a cobertura do plano de saúde privado, menor a quantidade de internações por condições sensíveis.
Em um outro estudo ${ }^{8,31}$, foi indicado que os territórios que apresentam um número mais elevado de leitos privados em relação aos disponibilizados pelo SUS tendem a diminuir a internação nestes leitos públicos. Ou seja, esse dado indica que as ICSAP em lugares com quantidades altas de leitos privados podem ser mais elevadas do que, de fato, os números indicam, visto que estas podem estar ocorrendo nesses outros espaços. Assim, a cobertura dos planos de saúde não estão, necessariamente, trazendo mais acesso e qualidade de atenção às questões enfocadas pela $\mathrm{AB}$, mas podem, ao contrário, estar ocultando essas internações, já que os dados referentes à prestação de serviços em âmbito particular não estão disponíveis para acesso público.

É compreensível, portanto, que as ICSAP apresentem uma menor incidência nos municípios com cobertura mais ampla dos planos de saúde. Contudo, é importante apontar que esse fato não indica, necessariamente, uma melhora no acesso e na qualidade da atenção do ponto de vista da promoção de saúde e da prevenção de doenças, isso porque as ICSAP podem, ainda, estar ocorrendo, mas em ambiente privado; diminuindo, nesse caso, a contabilização destas nos leitos do SUS.

Ao observar a variável referente à disponibilidade de leitos, nota-se, com exceção da região Nordeste, que, nas outras regiões, esse valor se relacionou de forma positiva com a variável dependente, indicando que quanto mais leitos, maior o número de ICSAP. Nesse sentido, cabe destacar que a disponibilidade de serviços de saúde pode induzir a utilização destes por uma determinada população. De forma oposta, a oferta pode ocorrer por influência do uso, formando um ciclo que se retroalimenta ${ }^{31}$. Ao considerar que pode existir esse estímulo à utilização dos leitos em situações que aparentemente não necessitariam, isto é, por simples indução à utilização por conta da oferta, o sistema de saúde estaria não só aumentando o número de internações desnecessárias, mas também impedindo as pessoas que realmente precisam de atendimento acessarem estes 
serviços. Assim, o número elevado de utilização dos leitos, nessa situação, não está relacionado, necessariamente, com uma melhora na qualidade dos serviços ${ }^{\mathbf{8}, 31}$; sendo necessários, em relação à região Nordeste, novos estudos visando compreender o cenário oposto em relação a essa variável.

Foi possível observar, também, que a cobertura da $A B$ não apresentou uma influência significativa ao verificar as taxas de incidência das ICSAP durante os anos de 2010 a 2014. Tal questão pode estar conectada com o fato de essa atenção ter passado por sua maior expansão durante os últimos anos, indo de $6,55 \%$ para $64,72 \%{ }^{32}$. Assim, esta passou a apresentar valores mais estáveis durante a pesquisa, visto que o seu crescimento acentuado ocorreu nos anos anteriores.

Ao considerar a variável relacionada com o programa Mais Médicos, ressalta-se um estudo $^{8}$ que observou a proporção de ICSAP em relação ao total de internações por outras causas no ano de 2012. Os resultados apontaram um declínio dessa proporção no Brasil, apresentando variáveis, como a oferta de médico nos municípios, associadas com a diminuição das ICSAP. Esse último efeito ocorreu em graus distintos nas regiões brasileiras, levando o autor a concluir que a distribuição adequada de médicos se configura um dos fatores importantes para aperfeiçoar a AB. Considerando esse argumento, é possível notar que um dos objetivos do programa Mais Médicos é justamente contribuir para diminuir a escassez de médicos em determinadas regiões do Brasil. Assim, parece ficar clara a importância dessa política no aperfeiçoamento da $A B$, uma vez que a influência dessa variável na diminuição das ICSAP foi considerável.

Por último, é importante considerar os determinantes socioeconômicos ao analisar os distintos efeitos do PMAQ nas regiões brasileiras ${ }^{6-8,11,33-35}$, dado que estes podem estar relacionados com as condições de saúde da população ${ }^{36}$. Durante o período de 2001 a 2012, abarcando, portanto, uma parte dos anos analisados neste estudo, o Brasil presenciou um crescimento no Produto Interno Bruto (PIB) nacional. Nesse contexto, a região Nordeste apresentou o aumento mais elevado ${ }^{36}$. Além disso, a taxa de analfabetismo reduziu, apresentando a região supracitada, novamente, como o local onde esse cenário ocorreu de forma mais acentuada ${ }^{36}$. Essa situação, em conjunto com a diminuição da proporção de pessoas com baixa renda em todo o Brasil e a saída de 22 milhões de pessoas da condição de extrema pobreza $^{36}$, aponta para uma melhora nas condições socioeconômicas do País. Nesse sentido, o avanço positivo significativo dos indicadores socioeconômicos pode ter contribuído, também, para a melhora da saúde da população e diminuição das ICSAP ${ }^{37-39}$ no Brasil e para o destaque da região Nordeste.

Esta seção do artigo procurou elucidar o cenário exposto pelos resultados aqui apresentados, a saber, a heterogeneidade do efeito do PMAQ nas ICSAP, considerando as regiões brasileiras. Essa questão parece estar sendo respondida por meio da discussão realizada com a literatura referente à temática deste trabalho ao notar que as regiões apresentaram características distintas em relação às ações, aos recursos e aos estabelecimentos na área da saúde, às dinâmicas socioeconômicas e às questões conjunturais inerentes a cada lugar. Nesse sentido, esses aspectos podem estar conectados com os distintos graus de melhora do acesso e da qualidade da $A B$ das regiões; sendo imprescindível, nesse caso, para a análise aprofundada do efeito do programa nesses lugares, considerar distintos fatores.

Por fim, os dados aqui expostos, de forma geral, confirmam a tendência de queda das ICSAP no País mostradas nos estudos que utilizaram esse indicador $6,8,10,40,41$. Além disso, corrobora a literatura ao expor os distintos cenários em relação às taxas de ICSAP nas regiões brasileiras ${ }^{\mathbf{8}, \mathbf{4 1}}$. A diminuição das internações aqui estudadas, demonstrada neste artigo a partir do PMAQ, vai em direção aos estudos que confirmam a importância 
das equipes da $\mathrm{AB}$ para a sua redução ${ }^{10,33,41}$. A partir da discussão, parece imprescindível considerar que existem aspectos intrínsecos a cada região que podem ter influenciado o alcance do efeito do programa, sendo importante, assim, considerando os resultados obtidos a partir do indicador ICSAP, observar os serviços de saúde disponíveis e os determinantes socioeconômicos de cada local, visto que estes podem facilitar ou obstaculizar a utilização dos dispositivos de saúde 6-8,11,33-35. Os resultados alcançados neste estudo fortalecem a importância do PMAQ e reafirmam as ICSAP como um indicador capaz de avaliar o acesso e a qualidade da AB; contribuindo, assim, para a produção acadêmica sobre essa temática e destacando a utilidade desse indicador para avaliar os efeitos de políticas públicas da $\mathrm{AB}$ na saúde da população.

\section{Considerações finais}

A partir dos resultados obtidos, indo ao encontro da hipótese elencada, foi possível concluir que o PMAQ está atingindo o seu objetivo em aumentar o acesso e a qualidade da AB. Entre as diferentes perspectivas possíveis para observar esses aspectos, neste trabalho, foi utilizado o indicador 'ICSAP'. Em relação a este, é importante, considerando os resultados, pontuar os seus possíveis limites ao avaliar o PMAQ. Como discutido neste artigo, existem aspectos distintos em cada região do País que podem facilitar ou obstaculizar a utilização dos dispositivos de saúde. Nesse sentido, destaca-se não só a importância das ICSAP para a avaliação do PMAQ, mas também outros fatores contextuais que devem ser ponderados, em conjunção com esse indicador, ao analisar esse programa.

Além disso, cabe destacar novamente as limitações referentes ao número de equipes que participaram do PMAQ, especialmente no primeiro ciclo, visto que foi estabelecido um limite máximo de equipes que poderiam aderir ao programa; a utilização do valor do coeficiente de Gini de 2010 para todos os anos analisados; e a possível limitação, por parte dos órgãos responsáveis, na coleta de dados municipais utilizados na pesquisa; exigindo, dessa forma, cautela ao analisar os efeitos do PMAQ nas ICSAP.

Por fim, destaca-se que as distintas realidades regionais existentes no Brasil na área da saúde demandam cada vez mais o fortalecimento de políticas públicas que considerem as características locais. Tal exigência clama pela atuação conjunta das três entidades de governo - a União, os estados e os municípios -, uma vez que só com a cooperação será possível atingir um leque diferenciado de políticas que abarquem as necessidades de cada localidade, mas que mantenha, em nível nacional, uma equidade na distribuição desses serviços. O processo de avaliação se apresenta nesse contexto como um instrumento responsável por iluminar o caminho que se está seguindo, permitindo que ajustes sejam feitos para alcançar a qualidade e a equidade no acesso aos serviços de saúde. Nesse contexto, este artigo surge como um importante informativo sobre as políticas públicas de saúde no Brasil e, também, como um meio de instrumentalizar os gestores e os diversos atores envolvidos no desenvolvimento e discussão de um dos principais programas da AB: o PMAQ.

Sugere-se que os novos estudos sobre a temática discutida neste artigo abordem os efeitos do PMAQ nas ICSAP explorando, em detalhes, a faixa etária, o sexo e as doenças que causaram as internações, posto que essas informações podem contribuir para a compreensão da influência de fatores socioeconômicos e ambientais na saúde da população que extrapolam a ação da $\mathrm{AB}$ - informação importante para analisar os limites e possibilidades do PMAQ, assim como o alcance do indicador ICSAP ao se propor avaliar indiretamente o acesso e a qualidade da AB. 


\section{Agradecimentos}

Os autores do artigo agradecem ao Professor

Dr. Sérgio Luiz Bassanesi (in memoriam) pelas importantes sugestões e comentários na elaboração do conteúdo deste artigo.

\section{Referências}

1. Brasil. Ministério da Saúde. Departamento de Atenção Básica. [internet]. [acesso em 2018 dez 10]. Disponível em: http://dab.saude.gov.br/portaldab/pnab.php.

2. Starfield B, Shi L, Macinko J. Contribution of Primary care to health systems and health. MIQUES. 2005; 83(3):457-502.

3. Mendes EV. A Construção Social da Atenção Primária à Saúde. Brasília, DF: CONASS; 2015.

4. Donabedian A. Aspects of medical care administration. Boston: Harvard University Press; 1973.

5. Donabedian A. The seven pillars of quality. Arch Pathol Lab Med. 1990; 114:1115-1118.

6. Alfradique ME, Bonolo PF, Dourado I, et al. Internações por condições sensíveis à atenção primária: a construção da lista brasileira como ferramenta para medir o desempenho do sistema de saúde (Projeto ICSAP - Brasil). Cad. Saúde Pública. 2009; 25(6):133749.

7. Nunes KR. Análise das internações por condições sensíveis à Atenção Primária em hospital de referência regional [tese]. Botucatu: Universidade Estadual Pauand Contributor ID).

\section{Colaboradores}

Soares C (0000-0001-7833-2695)* e Ramos M (0000-0003-0880-8269)* contribuíram igualmente para a elaboração do manuscrito.
8. Castro ALB, Andrade CLT, Machado CV, et al. Condições socioeconômicas, oferta de médicos e internações por condições sensíveis à atenção primária em grandes municípios do Brasil. Cad. Saúde Pública. 2015 ; 31(11):2353-66.

9. Cardoso CS, Pádua CM, Rodrigues-Júnior AA, et al. Contribuição das internações por condições sensíveis à atenção primária no perfil das admissões pelo sistema público de saúde. Rev. Panam Salud Publica. 2013; 34(4):227-34.

10. Carvalho SC, Mota E, Dourado I, et al. Hospitalizations of children due to primary health care sensitive conditions in Pernambuco State, Northeast Brazil. Cad. Saúde Pública. 2015; 31(4):744-54.

11. Melo MD, Egry EY. Determinantes sociais das Internações por Condições Sensíveis à Atenção Primária em Guarulhos, São Paulo. Rev. esc. enferm. USP. 2014; 489(esp):129-36.

12. Pereira FJR, Silva CC, Lima Neto EA. Condições Sensíveis à Atenção Primária: uma revisão descritiva dos resultados da produção acadêmica brasileira. Saúde debate. 2014; 38(esp):331-42.

13. Oliveira VB. Avaliação do Impacto das Ações do 
Programa de Saúde da Família na Redução das Internações Hospitalares por Condições Sensíveis à Atenção Básica em Adultos e Idosos. [internet]. BH: NESCON; 2012. [acesso em 2020 ago 4]. Disponível em: https://www.nescon.medicina.ufmg.br/biblioteca/imagem/3261.pdf.

14. Brasil. Ministério da Saúde. Sistema de Informações Hospitalares: DataSUS (SIH-DATASUS). [internet]. [acesso em 2018 nov 8]. Disponível em: http://sihd. datasus.gov.br/principal/index.php.

15. Brasil. Ministério da Saúde. Departamento de Atenção Básica: PMAQ (DAB-PMAQ). [internet]. [acesso em 2018 dez 10]. Disponível em: http://dab.saude. gov.br/portaldab/ape_pmaq.php.

16. Brasil. Ministério da Saúde. Indicadores de Saúde e Pactuações: DataSUS (PACTUAÇÕES-DATASUS). [internet]. [acesso em $2018 \mathrm{dez} 10$ ]. Disponível em: http://www2.datasus.gov.br/DATASUS/index. php?area $=0201$.

17. Brasil. Ministério da Saúde. Cadastro Nacional de Estabelecimentos de Saúde (CNES-DataSUS). [internet]. [acesso em 2018 dez 10]. Disponível em: http:// cnes.saude.gov.br/pages/downloads/arquivosBaseDados.jsp.

18. Instituto Brasileiro de Geografia e Estatística. População. [internet]. [acesso em 2019 jan 3]. Disponível em: https://www.ibge.gov.br/estatisticas-novoportal/sociais/populacao.html.

19. Agência Nacional de Saúde Suplementar. Dados e indicadores de setor [internet]. [acesso em 2019 jan 10]. Disponível em: http://www.ans.gov.br/perfil-do-setor/dados-e-indicadores-do-setor.

20. Programa das Nações Unidas para o Desenvolvimento. Atlas de desenvolvimento humano do Brasil. [internet]. [acesso em $2018 \mathrm{dez} 2$ 2]. Disponível em: http:// atlasbrasil.org.br/2013/pt/download/.

21. Região e Redes: Caminho da universalização da saúde no Brasil. [internet]. [acesso em 2019 jan 5]. Disponível em: http://www.resbr.net.br/indicadores/view/.
22. Brasil. Programa Mais Médicos (SPMM). [internet]. [acesso em 2019 jan 5]. Disponível em: http://maismedicos.gov.br/conheca-programa.

23. Universidade da Califórnia em Los Angeles. Data Analysis Examples. [internet]. [acesso em 2019 jan 10]. Disponível em: https://stats.idre.ucla.edu/other/ dae/.

24. Cavalcanti PCS, Oliveira Neto AV, Silva LE, et al. Análise da Estrutura Física das Unidades Básicas de Saúde no Brasil. In: Gomes LB, Barbosa MG, Ferla AA, editores. Atenção básica: olhares a partir do programa nacional de melhoria do acesso e da qualidade (PMAQ-AB). Porto Alegre: Rede UNIDA; 2016. p. 127-160.

25. Facchini LA, Piccini RX, Tomasi E, et al. Desempenho do PSF no Sul e no Nordeste do Brasil: avaliação institucional e epidemiológica da Atenção Básica à Saúde. Ciênc. Saúde Colet. 2006; 11(3):669-81.

26. Moura BLA, Cunha RC, Fonseca ACF, et al. Atenção primária à saúde: estrutura das unidades como componente da atenção à saúde. Rev. Bras. Saúde Mater. Infant. 2010; 10(1):69-81.

27. Bousquat A, Giovanella L, Fausto MCR, et al. Tipologia da estrutura das unidades básicas de saúde brasileiras: os 5 R. Cad. Saúde Pública. 2017 [acesso em 2020 ago 4]; 33(8):e00037316. Disponível em: https://www. scielo.br/scielo.php?script=sci_arttext\&pid=S0102$-311 X 2017000805005$.

28. Araujo WRM. Internações por condições sensíveis à atenção primária no Brasil: análise hierárquica de fatores de contexto, estrutura e processo de trabalho [dissertação]. São Luís: Universidade Federal do Maranhão; 2015.

29. Neves TCC, Montenegro LAA, Bittencourt SDA. Produção e registro de informações em saúde no Brasil: panorama descritivo através do PMAQ-AB. Saúde debate. 2014; 38(103):756-70.

30. Bahia L, Scheffer M. Planos e Seguros Privados de Saúde. In: Giovanella L, Escorel S, Lobato LVC, et al., 
editores. Políticas e sistemas de saúde no Brasil. Rio de Janeiro: Fiocruz; 2012. p. 1.636-1.656.

31. Travassos C, Castro MSM. Determinantes e Desigualdades Sociais no Acesso e na Utilização de Serviços de Saúde. In: Giovanella L, Escorel S, Lobato LVC, et al., editores. Políticas e sistemas de saúde no Brasil. Rio de Janeiro: Fiocruz; 2012. p. 677-766.

32. Cavalcanti PCS, Oliveira Neto AV, Sousa MF. Uma Narrativa Sobre o Programa Nacional de Melhoria do Acesso e da Qualidade na Atenção Básica. In: Gomes LB, Barbosa MG, Ferla AA, editores. Atenção básica: olhares a partir do programa nacional de melhoria do acesso e da qualidade (PMAQ-AB). Porto Alegre: Rede UNIDA; 2016. p. 17-48.

33. Nunes RP. Estratégia Saúde da Família e Internações por Condições Sensíveis à Atenção Primária: uma revisão sistemática. APS; 2018; 21(3):450-60.

34. Pinto Junior EP, Aquino R, Medina MG, et al. Efeito da Estratégia Saúde da Família nas internações por condições sensíveis à atenção primária em menores de um ano na Bahia, Brasil. Cad. Saúde Pública [internet]. 2018 [acesso em 2020 ago 4]; 34(2):e00133816. Disponível em: https://www.scielo.br/scielo. php?script=sci_abstract\&pid=S0102-311X2018000 205003\&lng=es\&nrm=iso\&tlng=pt.

35. Barros FC, Matijasevich A, Requejo JH, et al. Recent trends in maternal, newborn, and child health in Brazil: progress toward Millennium Development Goals 4 and 5. Am J Public Health. 2010; 100(10):1877-89.

36. Brasil. Ministério da Saúde. Plano Nacional de Saúde: PNS, 2016-2019. [internet]. [acesso em 2020 maio 28]. Disponível em: http://bibliotecadigital.seplan. planejamento.gov.br/handle/123456789/1048?sho $\mathrm{w}=$ full.

37. Bettenhausen JL, Colvin JD, Berry JG, et al. Association of Income Inequality With Pediatric Hospitalizations for Ambulatory Care-Sensitive Conditions. JAMA Pediatr [internet]. 2017 [acesso em 2020 ago 4]; 171(6):e170322-el70322. Disponível em: https:// jamanetwork.com/journals/jamapediatrics/fullarticle/2613462.

38. Roos LL, Dragan R, Schroth RJ. Pediatric ambulatory care sensitive conditions: Birth cohorts and the socio-economic gradient. Can J Public Health. [internet]. 2017 [acesso em 2020 ago 4]; 108(3):e257-e264. Disponível em: https://pubmed.ncbi.nlm.nih. gov/28910247/.

39. Agha MM, Glazier RH, Guttmann A. Relationship between social inequalities and ambulatory caresensitive hospitalizations persists for up to 9 years among children born in a major Canadian Urban Center. Ambulatory Pediatrics. 2007; 7(3):258-262.

40. Ribeiro MGC, Araujo Filho ACA, Rocha SSD. Hospitalizações por condições sensíveis à atenção primária em crianças do Nordeste Brasileiro. Rev. Bras. Saude Mater. Infant. 2019; 19(2):491-498.

41. Pinto LF, Giovanella L. Do Programa à Estratégia Saúde da Família: expansão do acesso e redução das internações por condições sensíveis à atenção básica (ICSAB). Ciênc. Saúde Colet. 2018; 23:1903-1914.

Recebido em 04/10/2019

Aprovado em 10/06/2020

Conflito de interesses: inexistente

Suporte financeiro: não houve 